\title{
Letter: Non-Nutritive Sweeteners May Have a Bell-Shaped Dose Response for Dysbiosis-Related Adverse Effects That Contributed to Discrepancies between Prospective Cohort and Randomized Controlled Studies
}

\author{
Xiaofa Qin* \\ GI Biopharma Inc., Westfield, NJ, USA
}

I read with great interest the recent paper by Walbolt and $\mathrm{Koh}^{1}$ regarding the effects of non-nutritive sweeteners (NNS) on obesity and type 2 diabetes. It reported that most prospective cohort studies found positive association between NNS and obesity or diabetes, while many of the randomized controlled studies showed neutral or even negative association. It provided some explanations such as the effect of the sweet taste of NNS on appetite and food intake or potential increased selection of NNS by people with obesity or diabetes in the prospective cohort that caused reverse causality. However, as discussed in the paper, increased weight gain with reduced food intake in livestock fed NNS suggested the existence of a true adverse effect of NNS on obesity and its closelyassociated diabetes. Here, I would like to provide more explanations for discrepancies between the prospective cohort and randomized controlled studies using more detailed analyses.

Increased growth rate and weight gain in many livestock, including cows, pigs, sheep, and chickens, have been shown since the 1950s after treatment with a wide range of antibiotics at subtherapeutic low dose but not at therapeutic high dose. ${ }^{2}$ For instance, $70 \mathrm{mg} / \mathrm{animal} /$ day may be used for growth promotion in cattle, while $6,600 \mathrm{mg} / \mathrm{animal} / \mathrm{day}$ may be needed to treat an infection. ${ }^{2}$ This bell-shaped dose response was also seen in many other studies. Abou-Donia et al. ${ }^{3}$ treated groups of mice with $1.1-$ $11 \mathrm{mg} / \mathrm{kg} /$ day sucralose for 12 weeks. The most pronounced significant increase in body weight was seen in the $1.1 \mathrm{mg} / \mathrm{kg} / \mathrm{day}$ sucralose group, along with changes in gut bacteria. In addition, rats treated with $0.0005 \%(0.005 \mathrm{mg} / \mathrm{mL})$ saccharin in drinking water for 6 weeks showed significant high blood glucose and lower food intake but higher weight gain; ${ }^{4}$ rats treated with $0.1 \mathrm{mg} / \mathrm{mL}$ (0.01\%) saccharin for 7 weeks had increased blood glucose but no difference in body weight; ${ }^{5}$ while rats treated with $1.0 \%-7.5 \%$ saccharin showed a dose-dependent decrease in body weight. ${ }^{6}$

More importantly, this bell-shaped dose-response also was shown in randomized controlled clinical trials in humans. A study by Bueno-Hernández et al. ${ }^{7}$ showed that healthy volunteers receiving $48 \mathrm{mg}$ sucralose daily for 10 weeks had significantly higher levels of blood insulin and glucose during an oral glucose tolerance test and reduced insulin sensitivity, while the $96 \mathrm{mg}$ sucralose daily group failed to show such effects. The randomized controlled clinical trial by Romo-Romo et al. ${ }^{8}$ found that healthy 
adults ingesting $36 \mathrm{mg}$ sucralose daily for 2 weeks had a significant decrease in insulin sensitivity. In contrast, a double-blind, placebo-controlled, randomized clinical trial showed no effect on insulin sensitivity or glucose tolerance in healthy subjects who consumed $1 \mathrm{~g}$ sucralose daily for 12 weeks. ${ }^{9}$ Together, these well designed clinical trials suggest the existence of a more potent adverse effect of low-dose sucralose than high dose.

How to explain the bell-shaped pattern of dose response? As discussed in the paper by Walbolt and Koh, ${ }^{1}$ the critical role of the gut microbiome on health has drawn increasing attention. Studies have well documented common changes in dysbiosis-associated health problems, such as decreases in abundance and diversity of gut bacteria, increased gut permeability, and systemic, low-grade inflammation and endotoxemia. Here, I propose that the bell-shaped response to NNS shares a similar mechanism to the low incidence of inflammatory bowel disease in both poor hygiene and germ-free conditions that may result from degradation of the protective mucus layer. ${ }^{10}$

The mucus layer is a crucial component of the gut barrier, and mucin, the key component of mucus, is a glycoprotein that contains a core peptide and many (up to $90 \%$ in weight) side carbohydrate chains. Thus, degradation of mucus requires both proteases, which mainly originate from the pancreas, to break down the peptide, and glycosidases, which come mainly from gut bacteria, to break down carbohydrates. Under conventional situations, digestive proteases are promptly inactivated by bacteria to prevent rapid degradation of protective mucus. A reduction in gut bacteria by NNS, such as saccharin or sucralose, may lead to impaired inactivation of digestive proteases that may work synergistically with bacterial glycosidases, resulting in accelerated degradation of the mucus layer. However, further reduction in gut bacteria may reach a point at which degradation will start to slow down due to great reduction in bacterial glycosidases. In addition, further reduction in gut bacteria would also reduce the amount and availability of bacterial lipopolysaccharides penetrating the body. These may result in decreases in endotoxemia and systemic inflammation and, thus, the potency of related adverse effects.

Randomized controlled studies showed potent adverse effects of sucralose on body weight and blood glucose at doses of $48 \mathrm{mg}$ or $36 \mathrm{mg}$ daily, an intake less than $1 \mathrm{mg} / \mathrm{kg} / \mathrm{day}$ and a small frac- tion of the $5 \mathrm{mg} / \mathrm{kg} /$ day acceptable daily intake (ADI) set by the Food and Drug Administration in the United States and the 15 $\mathrm{mg} / \mathrm{kg}$ /day ADI set by the Joint Food and Agriculture Organization and the World Health Organization Expert Committee in Food Additives. Thus, the peak of the most potent adverse effect may be much lower than the ADI, which may be closer to the dose consumed by the general population in the prospective cohorts. In contrast, the doses used in many randomized controlled trials may have far surpassed these most potent doses, which could contribute to discrepancies between prospective cohort and randomized control studies. NNS may have more potent adverse effects on health than are currently perceived. I suggest conducting more studies in this regard.

\section{CONFLICTS OF INTEREST}

The author declares no conflict of interest.

\section{REFERENCES}

1. Walbolt J, Koh Y. Non-nutritive sweeteners and their associations with obesity and type 2 diabetes. J Obes Metab Syndr 2020;29:114-23.

2. Cox LM. Antibiotics shape microbiota and weight gain across the animal kingdom. Anim Front 2016;6:8-14.

3. Abou-Donia MB, El-Masry EM, Abdel-Rahman AA, McLendon RE, Schiffman SS. Splenda alters gut microflora and increases intestinal p-glycoprotein and cytochrome p-450 in male rats. J Toxicol Environ Health A 2008;71:1415-29.

4. Andrejić BM, Mijatović VM, Samojlik IN, Horvat OJ, Ćalasan JD, Đolai MA. The influence of chronic intake of saccharin on rat hepatic and pancreatic function and morphology: gender differences. Bosn J Basic Med Sci 2013;13:94-9.

5. Leibowitz A, Bier A, Gilboa M, Peleg E, Barshack I, Grossman E. Saccharin increases fasting blood glucose but not liver insulin resistance in comparison to a high fructose-fed rat model. Nutrients 2018;10:341.

6. Schoenig GP, Goldenthal EI, Geil RG, Frith CH, Richter WR, Carlborg FW. Evaluation of the dose response and in utero exposure to saccharin in the rat. Food Chem Toxicol 1985;23: 
475-90.

7. Bueno-Hernández N, Esquivel-Velázquez M, Alcántara-Suárez R, Gómez-Arauz AY, Espinosa-Flores AJ, de León-Barrera KL, et al. Chronic sucralose consumption induces elevation of serum insulin in young healthy adults: a randomized, double blind, controlled trial. Nutr J 2020;19:32.

8. Romo-Romo A, Aguilar-Salinas CA, Brito-Córdova GX, Gómez-Díaz RA, Almeda-Valdes P. Sucralose decreases insulin sensitivity in healthy subjects: a randomized controlled trial.
Am J Clin Nutr 2018;108:485-91.

9. Grotz VL, Pi-Sunyer X, Porte D Jr, Roberts A, Richard Trout J. A 12-week randomized clinical trial investigating the potential for sucralose to affect glucose homeostasis. Regul Toxicol Pharmacol 2017;88:22-33.

10. Qin X. Synergic effect of bacterial glycosidases and digestive proteases on mucus degradation and the reduced risk of inflammatory bowel disease-like gut damage in both germ-free and poor hygiene conditions. Inflamm Bowel Dis 2008;14:145-6. 